\title{
An automated device for measuring locomotor activity in rats
}

\author{
P. E. SCHENCK, H. VAN DE GIESSEN,* A. KOOS SLOB, \\ and J. J. VAN DER WERFF TEN BOSCH \\ Department of Endocrinology, Growth and Reproduction and "Central Research Workshop \\ Faculty of Medicine, Erasmus University, Rotterdam, The Netherlands
}

An automated plus maze is described that is capable of recording the locomotor activity of 12 rats simultaneously. The system is built around an 8008 microprocessor chip from INTEL. The base of the system is formed by 12 plus mazes in which the activity of the animals under test can be monitored.

A classical instrument for measuring locomotor activity of rats is the running wheel or treadmill. According to Barnett (1963), this apparatus enhances animal activity because "the presence of a wheel, or at least its movement once running has begun, may act as a stimulus to further running." It is sometimes desirable to have an instrument that does not stimulate the animal's locomotor activity, and the residential plus maze seems to fulfill this requirement (Barnett, Cockroft, \& Smart,

The research was financially supported by the Foundation for Medical Research, FUNGO, which is subsidized by the Netherlands Organization for the Advancement of Pure Research (Z. W. O.). Thanks are due Mrs. C. Borg, Miss J. van der Gaag, Mr. C. Geilman, Miss A. Bot, and Mrs. P. Landsmeer for their help in analyzing the data, critical reading, and typing the manuscript.
1966; Barnett, Smart, \& Widdowson, 1971). This paper contains a description of an automated modified version and its validation with normal cycling female rats.

\section{DESCRIPTION OF THE APPARATUS}

Figure 1 shows a plan of the maze drawn to scale. Four arms extend from the central box to smaller terminal boxes. One terminal box (No. 1) contains two water bottles; another (No. 3) gives access to a food bin with powdered rat chow. Terminal boxes 2 and 4 are empty. The central box and the arms can be separated by guillotine doors, so that animals can be retained in the central box whenever necessary, as at the beginning and end of an experiment. The central boxes are covered by clear Plexiglas, and a $25 . \mathrm{W}$ white fluorescent tube is

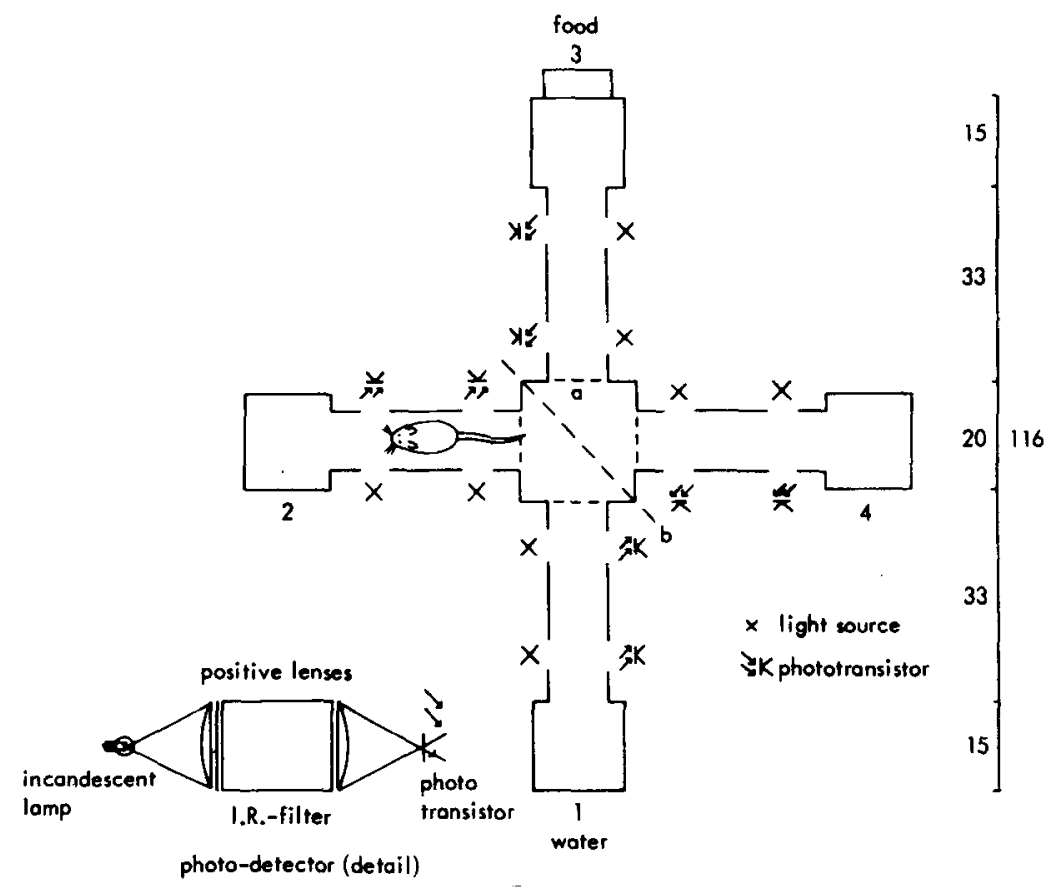

Figure 1. The plus maze: $a=$ site of guillotine door, $b=$ diagonally placed fluorescent tube. Scale in centimeters. 
fixed $40 \mathrm{~cm}$ from the bottom of the central box. Movement of the animal from the central box to the distal end of an arm and back is monitored by infrared light detectors. Two detectors are needed in each arm. In order to make the detector insensitive to the animal's tail, two lenses are added to the combination of lamp and phototransistor; an additional infrared filter makes the light source invisible to the animal.

\section{Interface}

To make the data suitable for computer processing, an interface developed for a paper-tape punch handles up to six mazes simultaneously. Data from the mazes are fed into amplifiers in order to generate enough signal strength to activate the Schmitt triggers, which convert the slow variations of the input signal to discrete TTL levels. The signals are latched and scanned by a highspeed scanner. If a latch is set by an event (arm in or arm out), the scanner stops and the event is punched, together with the arm and maze identification and the time in minutes and seconds. The hour is punched separately (after each hour transition) to obtain a higher data density on the paper tape. At the start of an experiment, an identification code can be punched by means of a thumbwheel switch and pushbutton.
Table 1

\begin{tabular}{lr}
\hline 1 CPU & imm 8-82 \\
1 RAM memory (4 bytes RAM) & imm 6-28 \\
1 PROM memory +3k bytes EPROM & imm 6-26 \\
2 Input/Output modules & imm 8-60 \\
1 user-designed module for signal level adaptation, \\
multiplexing, etc., power supplies, etc.
\end{tabular}

\section{Computer}

The punched data are fed into an 8008 computer (INTEL). The $8008 \mathrm{CPU}$ has 8-bit word length, an instruction set of 48 instructions (including conditional branching), binary arithmetic, logical, register-to-register and memory reference operations, and a machine cycle time of 12.5 microsec. The CPU is capable of addressing $16 \mathrm{k}$ bytes of memory.

Each INTELLEC 8 module system supplied by INTEL consists of a printed board $6.2 \times 8$ in. The computer is built around the modules shown in Table 1. Figure 2 supplies a block diagram of the computer.

The CPU module contains a provision to interrupt the processor. This is used for system start-up. The RAM memory module contains $4 \mathrm{k}$ bytes of memory, using INTEL's $21021 \mathrm{k}$ static RAM with a cycle time of 900 nsec. The PROM memory module provides

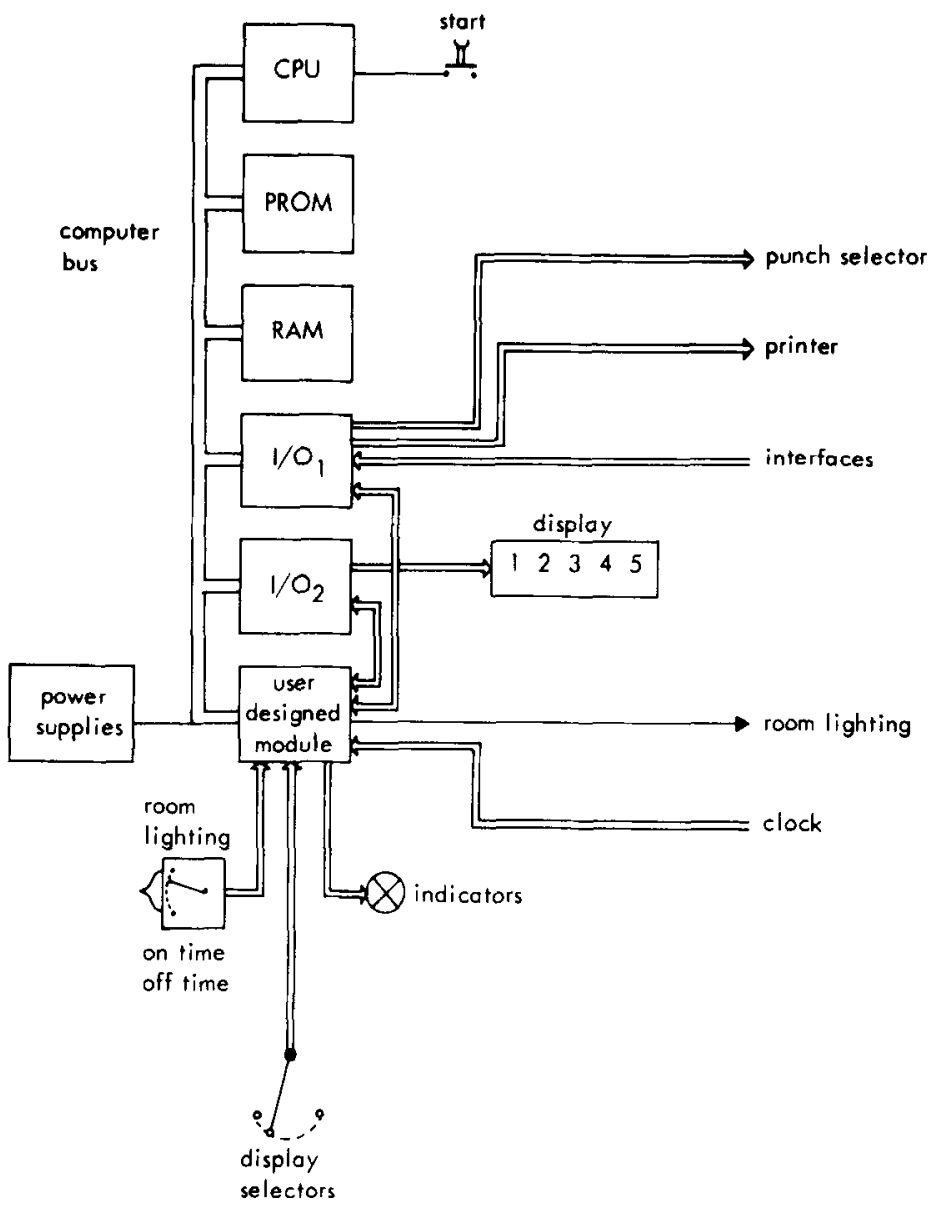

Figure 2. Computer block diagram. 
sockets for up to $161702 \mathrm{~A}$ EPROMS. The $1702 \mathrm{~A}$ is a $256 \times 8$ erasable programmable ROM. Erasing is done by exposing the EPROM to ultraviolet light through a transparent lid in the package. The $\mathrm{I} / 0$ module provides four 8-bit TTL-compatible input ports and four 8-bit TTL-compatible latching output ports. To cope with a limited number of input ports, the data from the clock and thumbwheel switches is multiplexed on a userdesigned module. This module also contains the lamp drivers for the indicators on the front panel of the computer. The computer is powered by $+5 \mathrm{~V}$ and $-9 \mathrm{~V}$.

\section{Peripherals}

Response data are printed every hour on a matrix printer, FACIT 4553. Intermediate results are displayed on a solid state display on the front panel of the computer; the maze and arm can be selected by switches. For additional processing, three FACIT 4070 paper-tape punches are available, one punch for each set of six mazes and one stand-by. The punches are connected to a punch selector, which automatically activates the standby punch in case the active punch runs out the paper tape. With the clock of one of the interface devices as reference, the processor switches the lighting of the plus maze room on and off at certain times. Times can be selected with thumbwheel switches on the processor's front panel. A block diagram of the system is shown in Figure 3.

\section{Representation of the Results}

The printer automatically prints the results of the running experiment: termination of an hour, termination of a $24 \mathrm{~h}$ period, and termination of the experiment when the total results are printed. A typical printout consists of a headline that lists an identification number of maximally eight digits, the type of printout, and the number of the hour or day. Then follows a list of the numbers of excursions per arm. An excursion

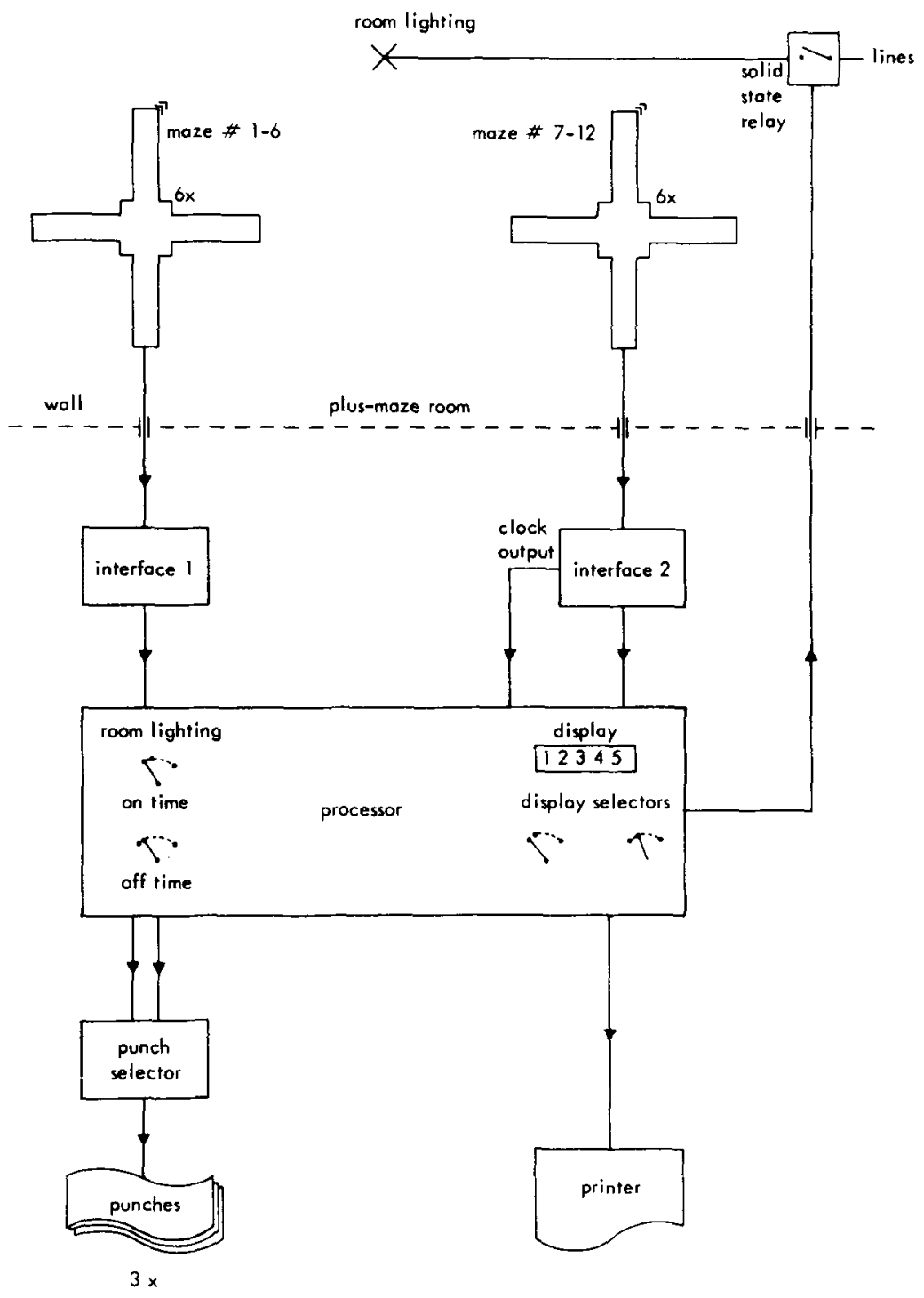

Figure 3. System block diagram. 
is a visit of the animal from the central box to the distal end and back. The listed quantities are in fact the number of excursions completed in the specified period. Next is printed a list with the total duration of visits to each arm over the specified period. The printing is completed with a list of the average duration per visit, that is, the total duration of visits divided by the number of excursions. As the experiment is terminated (by entering a new identification number), the processor produces the results of the ongoing hour, the ongoing day, and the total experiment. After that the processor is ready for collecting the data from a new experiment. Intermediate results of the ongoing experiment can be retrieved from the solid state display on the processor's front panel. Only the number of excursions of the ongoing hour, day, and experiment can be displayed.

\section{Program}

The program is written in assembly language and occupies $3 \mathrm{k}$ bytes of EPROM. There is no need to load the program every time the processor is switched on, so there is no bootstrap device. Because of poor interrupt capabilities of the 8008 microprocessor (complicated status saving), the interrupt is used only for initialization of the system. The peripherals are scanned rather than handled with interrupts. The program is developed with the aid of a cross-assembler, resident on a PDP-11/E10 minicomputer, which provides additional service as text editor. PROMS are programmed by a programmer controlled by another 8008 microprocessor. Special software was developed for checkout of the $\mathrm{I} / \mathrm{O}$ and the peripherals.

After receiving an interrupt request, caused by pressing the START button, the processor enters an initialization routine, then a scan loop. Successively, the processor collects data (if available) from the interfaces and continues with the handling of the display, the control of the room lighting, and the printing. Data from the interfaces are checked for validity (to avoid false results). The display routine inputs the status of the front panel switches and fetches the required data from the RAM, converts it to the decimal system, and sets the display according to the results. Room lighting is controlled by a solid state relay to avoid interference from switching transients. The printing routine checks the availability of print data. While printing is in progress, the printer is checked for readiness before each character is fed to the printer.

\section{SUPPORTING EXPERIMENT}

An experiment was carried out to investigate whether rats in the plus maze would grow normally and show the well-known variations in locomotor activity coincident with the ovarian cycle. Four groups of six rats (Wistar, Amsterdam R strain, 13 weeks of age) were consecutively housed in plus mazes for periods of 3 weeks. Lights in

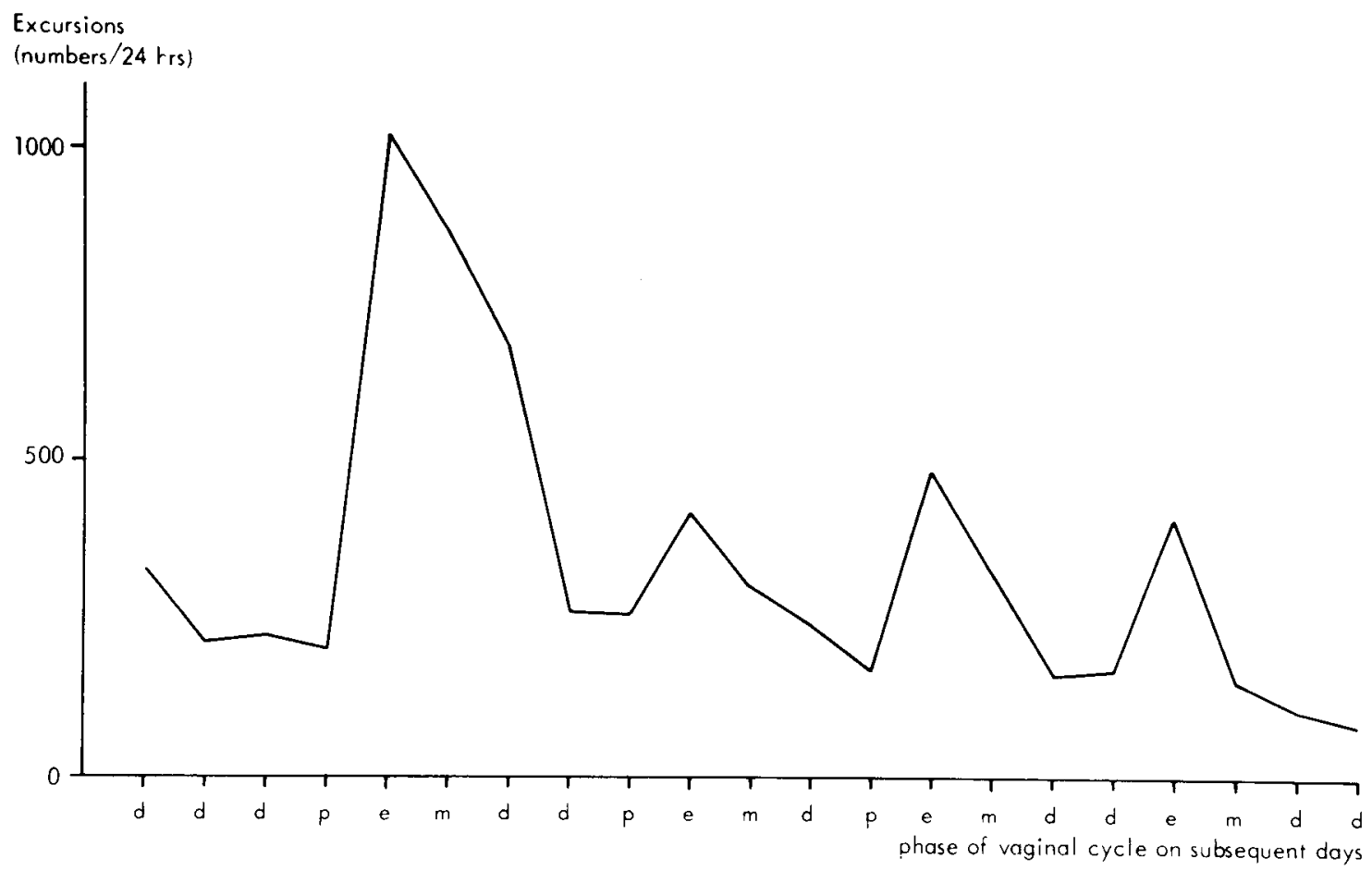

Figure 4. Locomotor activity in relation to the vaginal cycle of Rat 322, age: 19-22 weeks. Phases of the vaginal cycle are indicated at the bottom: $\mathrm{e}=$ estrus, $\mathrm{m}=$ metestrus, $\mathrm{d}=$ diestrus, $\mathrm{p}=$ proestrus. It is clear that the activity is highest at the times of estrus. 
the test room were on from $0900-2100 \mathrm{~h}$ and off from 2100-0900 h. A day encompassed the period from 0900 to $0900 \mathrm{~h}$. Six days a week, between 0900 and $1000 \mathrm{~h}$, the animals were taken out of the central boxes, weighed, and vaginal smears were taken. For each animal the mean activity score was computed for each phase of the cycle, i.e., a proestrus, an estrus, a metestrus, and a diestrus score. The daily scores were alotted to the cycle phase found at $0900 \mathrm{~h}$, that is, at the beginning of the day. The scores for identical phase days were averaged. One animal never showed a proestrus smear and was omitted from the analysis. The remaining 23 sets of four scores were subjected to the Friedman two-way analysis of variance.

Growth in body weight was studied over a period of 12 weeks. Weight increase slowed down with age over the 12-week period, and body weight was unaffected by residence in the plus maze. Vaginal cycles from subjects in the maze were followed for 3 weeks. Of the 101 cycles identified, there were 60 4-day cycles, 36 5-day cycles, and 5 6-day cycles. Mean locomotor activity (excursions/24 h) was 304 during estrus (E), 258 during metestrus (M), 213 during diestrus (D), and 208 during proestrus (P). Since overall analysis showed significant differences $\left(\chi^{2}=29.92, \mathrm{df}=3, \mathrm{p}<.001\right)$ in activity, these differences between cycle days were tested with the sign test. The activity on the day of vaginal estrus was significantly higher than in any other phase of the cycle $(p=.003$ for $E$ and $M ; p<.001$ for $E$ and $D$ and for $E$ and $P$ ). Activity at metestrus was higher than the activity displayed at diestrus $(p=.011)$. Figure 4 shows the effect of estrus cycles on locomotor activity of a single rat.

The normal growth in body weight and the regularity of the vaginal cycles indicate that residence in the plus maze is not harmful to the animals. Our finding that there is a relation between ovarian cycle and locomotor activity, the latter being highest during vaginal estrus, is in keeping with results of earlier studies with the running wheel (Wang, 1923) and with a "stationary cage" (Finger, 1961).

\section{REFERENCES}

BARnetT, S. A. The rat: A study in behavior. Chicago, Ill: Aldine, 1963.

Barnett, S. A., Cockroft, A. L., \& Smart, J. L. An artificial habitat for recording movement. Journal of Physiology, 1966. 187, 15P-16P.

Barnett, S. A., Smart, J. L., \& Widdowson, E. M. Early nutrition and the activity and feeding of rats in an artificial environment. Developmental Psychobiology, 1971, 4, 1-15.

FINGER, F. W. Estrous activity as a function of measuring device. Journal of Comparative and Physiological Psychology, 1961, 54, 524-526.

WANG, G. H. The relation between "spontaneous" activity and oestrus cycle in the white rat. Comparative Psychology Monographs, 1923, 6, 1-27.

\section{NOTE}

1. At the time the application of a microprocessor for processing the punched data came into question, the recording system had been in use for some time. The existing situation was that the paper tapes were sent to an IBM center, where they were processed by a previously made program, and the printer output was returned. This was time consuming and expensive. Therefore, the choice was made to install a microprocessor. At that time the reliability of microprocessors was not well known, so we decided to expand the system with a microprocessor without altering the existing setup. The hardware-scanning, buffering, real-time clock and paper-tape punch control were maintained, which was of course unnecessary if the whole job was done with a microprocessor.

Some functions were added, for example, switching the lights for artificial day and night and the possibility of displaying the contents of all the accumulating counters in the microprocessor during the experiment. Programming was in assembly language and took about 8 weeks. The system was tested without bothering the existing hardware system. A temporary paper-tape reader was hooked up to the microprocessor and an earlier produced paper tape was fed into the system. As long as the printed output was not exactly the same as the IBM output from that particular tape, the processing was incorrect and had to be debugged.

This testing procedure worked very well and, once the microprocessor was added to the system, there were only minor problems of installation. During programming, a simple crossassembler running on a PDP-11/10 was very helpful. A binary paper tape was produced and, with the help of an INTELLEC 8 system, a number of EPROMS were programmed.

(Received for publication August 26, 1977. Revision accepted April 10, 1978.) 